Published in final edited form as:

$J$ Adv Nurs. 2003 September ; 43(6): 631-641.

\title{
Preterm infants' behavioural indicators of oxygen decline during bottle feeding
}

Suzanne M. Thoyre, PhD RN [Assistant Professor] and

School of Nursing, University of North Carolina at Chapel Hill, Chapel Hill, North Carolina, USA

John R. Carlson, MA [Research Associate Professor]

School of Nursing, University of North Carolina at Chapel Hill, Chapel Hill, North Carolina, USA

\begin{abstract}
Background-During the time when preterm infants' oral feeding skills are developing they often experience physiological instability and need assistance from caregivers to maintain adequate oxygenation. Assisting infants to maintain optimal oxygenation during oral feeding requires an understanding of how they express and aim to self-regulate their oxygen status.
\end{abstract}

Aim-The purpose of this study was to identify potential behavioural indicators of declining oxygenation during preterm infant early bottle-feeding.

Method-The design was explorative. Data were collected from a secondary analysis of 20 videotapes of preterm infant bottle feedings which included concurrent oxygen saturation data. In this analysis infant behaviours and quality of breathing were coded and compared across three periods: high oxygen saturation, immediately preceding an oxygen desaturation event, and during an oxygen desaturation event.

Findings-Infants gave limited behavioural indicators of declining oxygenation. Immediately prior to a desaturation event, they had an increase in eye flutter and were typically sucking and apnoeic. During a desaturation event, they typically relaxed their arms/hands and stopped sucking.

Conclusions-Reliance on preterm infant behavioural cues will be insufficient for detection of oxygen desaturation during oral feeding. Attention to changes in breath sounds and to the pattern of sucking are potentially important intervention strategies for the prevention of and appropriate response to oxygen decline during feeding. Sucking pauses may be a time when preterm infants aim to regulate their breathing pattern and thereby increase oxygenation. Interventions that focus on detection and minimization of apnoea during feeding, and which aim to protect infant sucking pauses, may reduce the number and severity of desaturation events preterm infants experience during bottle feeding.

\section{Keywords}

preterm; oxygenation; desaturation; oral feeding; bottle feeding; sucking; energy; breathing; nursing

\section{Introduction}

During the weeks prior to discharge from neonatal intensive care units preterm infants are in the process of learning how to feed orally, at the breast and/or by bottle. This learning process 
is challenging, as preterm infants' sleep-wake states, postural control, oral-pharyngeal motor structures and control of breathing are often, in varying degrees, immature. Consequently, preterm infants are at risk for hypoxaemia during feeding, particularly when fed by bottle (Shiao et al. 1996, Poets et al. 1997a, Chen et al. 2000). Oxygen desaturation events during feeding may continue for several months after discharge, most significantly in the smallest and least healthy infants (Rosen et al. 1984, Garg et al. 1988, Singer et al. 1992). Despite common occurrences of hypoxaemia during bottle feeding, many infants are not monitored with pulse oximetry during feeding, particularly at home. Families and nurses must rely on visual monitoring of behaviour during feeding to direct support measures. However, little is known about the relationship between observable behaviour and oxygenation in the preterm infant, and an understanding of this relationship is required for the provision of appropriate feeding support. The purpose of this exploratory study was to examine whether specific observable behaviours or audible qualities of breathing during preterm infant bottle feeding can be used as behavioural indicators of declining oxygenation.

Feeding support, which includes detection and minimization of oxygen desaturation during feeding, is a highly important caregiver goal for preterm infants. Adequate oxygenation is critical for the growth and development of these infants (Moyer-Mileur et al. 1996, Cheung et al. 1999) and frequent desaturations, such as those that occur during feeding, may contribute to negative developmental and cognitive sequelae of prematurity (Perlman 2001). In addition, adequate oxygenation is thought to be the foundation for endurance, maintenance of body tone, oral-motor control, and coordination of sucking, swallowing, and breathing during feeding. Infants who have more oxygen desaturations during feeding have shorter sucking bouts and longer transition periods to full oral feeding (Shiao et al. 1996).

Providing sensitive and timely feeding support requires that families and nurses understand subtle infant behaviours that reflect their physiological state. Although this is not based on research, caregivers are advised to monitor and respond to signs of distress in the infant during feeding, including such behaviours as finger splay, arching, flaccidity, cyanosis and eyebrow raising (Vandenberg 1990, Als 1998, Shaker 1999). In response to these behaviours the caregiver may provide a rest period for the infant, change the infant's position, or tip the bottle back to stop milk flow and cue the infant to breathe. These subtle behaviours are often overlooked or misinterpreted by mothers of these babies during feeding. For example, in a study by Thoyre (1997), mothers interpreted their infants' pulling away from the nipple during a desaturation event as the infant being difficult, feeling full, or not liking the taste of the formula milk, when the infant may actually have been trying to breathe.

It is particularly important to understand preterm infant feeding behaviours because counterintuitive behaviour may be required of the caregiver. Very early work on mother-infant communication found that, when term infants paused in their sucking, it was common for mothers to jiggle the nipple to maintain the infant's attention to feeding (Kaye \& Wells 1980). Kaye and Wells described this behaviour as one of the first communication strategies in which mothers and infants engage. When the mother stopped jiggling, the infant resumed sucking. For preterm infants, jiggling may interfere with respiratory regulation, encourage milk to drip into the mouth of an infant needing to pause sucking in order to breathe, and thus may provide a new demand for the infant to adapt to. If nurses are to guide families toward appropriate responses to preterm infant's behavioural cues, particularly if this is counterintuitive (not jiggling during sucking pauses), we need to understand better the meaning of preterm infant behaviour during feeding. 


\section{Literature review}

The literature on the relationship of preterm infant behaviour with oxygen status during oral feeding was obtained by searching the CINAHL and PubMed databases, using the terms feeding and oxygen/oxygenation coupled with preterm infant breathing, sucking, and behaviour.

The breathing pattern of preterm infants during bottle feeding is highly variable (Craig et al. 1999). Breathing irregularities, including breathing pauses, often lead to significant and frequent episodes of desaturation during bottle feeding (Shiao et al. 1996, Chen et al. 2000). Mathew (1991) noted that recovery from respiratory dysregulation during feeding depends on the duration of breathing periods and ability of the infant to increase ventilation during these breathing breaks. In other words, the infant does not need merely to stop sucking at regular intervals for a breath, but must stop sucking and also take adequate quality breaths.

Balancing sucking bout lengths with an adequate number and quality of breaths is problematic for preterm infants. While term infants intersperse breaths between sucks and swallows, preterm babies typically alternate sucking and breathing with a series of sucks (sucking burst) followed by an interval of breathing (Bu'Lock et al. 1990). When sucking bursts are prolonged or breathing periods between sucking bouts are too short or interrupted, preterm infants may become hypoxaemic (Shiao 1997, Chen et al. 2000). Thoyre's (1997) finding that mean $\mathrm{SpO}_{2}$ per feeding episode was lower when preterm infants sucked more suggests that sucking may occur at the expense of respiration. Shiao et al. (1996), however, found that $97 \%$ of preterm infant desaturation events during feeding were accompanied by sucking pauses, pointing to an ability to engage in behaviour that is self-regulatory.

Both full-term and preterm infants typically engage in the longest sucking bursts at the outset of the feeding (Hill et al. 2000). During this 'continuous' sucking period, there is greater consumption of formula (Case-Smith et al. 1989, Hill 1992) and lower minute ventilation (less air moved per minute) because of lower respiratory frequency (Shivpuri et al. 1983, Mathew et al. 1985, Shiao 1997). Continuous sucking is followed by an 'intermittent' phase, in which sucking bursts shorten and the intervals between sucking bursts lengthen, thereby affording improved respiration. However, preterm infants often take brief, shallow breaths during the intermittent phase, and minute ventilation is recovered only partially (Shivpuri et al. 1983, Shiao 1997).

During the first 3 minutes of feeding, lower $\mathrm{SpO}_{2}$ is associated with increased loss of milk from the preterm infant's mouth (Hill \& Rath 1999). Hill and Rath speculate that during this early phase infants suck beyond their capacity to manage the bolus of fluid, become physiologically dysregulated and allow the excess milk to drool out of their mouths. In their study, the association of lower $\mathrm{SpO}_{2}$ with drooling did not remain during the final 3 minutes of the feeding; however, drooling behaviour was associated with diminished sucking during this period, perhaps an indication of muscle fatigue.

Changes in respiratory control may occur when infants' state of arousal decreases or when they become fatigued during feeding. Infants who spend more of their feeding time in an awake state have higher mean oxygen levels during the feeding (Thoyre 1997). Active sleep, the sleep state most likely if the infant falls asleep during feeding, depresses respiratory and arousal responses and many respiratory reflexes (Brouillette \& Côté 1996).

Adequate oxygenation enables infants to maintain behavioural organization (Porges 1994) and provides energy for the physiological work needed for effective and efficient feeding. Typically, infants feed in a flexed, toward midline, body position. When they begin to lose the ability to maintain this position (a loss of muscle tone) they may be showing fatigue. Less 
mature preterm infants demonstrate fatigue as feeding progresses (McGrath \& Medoff-Cooper 2002), as indicated by shorter sucking bursts and prolonged pauses between these bursts (Hill et al. 2000). The effect of fatigue on oxygenation during feeding has not been examined.

The capacity to respond behaviourally to a noxious event varies by postconceptional age (PCA), type of event encountered, and the infant's state during the encounter (Craig et al. 1993, Johnston et al. 1996, Grunau et al. 2000). Those with a PCA of 34-36 weeks, the age at which they are learning to feed orally, are capable of a full behavioural response to a painful procedure (Craig et al. 1993). Preterm babies who are more alert respond to noxious events more rapidly and with more facial activity than infants in quiet sleep states (Grunau \& Craig 1987). Additionally, the behavioural responses of those with bronchopulmonary dysplasia, a condition that is common among preterm infants learning to feed, are similar in those without lung disease (Holditch-Davis \& Lee 1993, Holditch-Davis 1995).

Two studies have examined specific infant behaviours in relation to oxygen status. Grunau et al. (2000) studied motoric movements in relation to endotracheal suctioning, a potentially distressing procedure that also has a negative impact on oxygenation. Infants were 24-32 weeks PCA at the time of the study and exhibited an increase in extensor behaviours during suctioning (finger splay, brow raising, leg extension). Peters (2001) examined the relationship between movement and physiological stability in infants between 28 and 32 weeks PCA during bathing. Extension behaviours consisting of finger splay, salute, airplaning and sitting on air occurred more frequently, either immediately prior to or during a decline in oxygenation. Flexion behaviours, consisting of hand to mouth, hand clasp, foot clasp, grasping and fisting, were more likely to occur during periods of increased $\mathrm{SpO}_{2}$ (Peters).

\section{The study}

Aim

The aim of this explorative study was to examine whether specific observable behaviours or audible qualities of breathing during preterm infant bottle feeding can be used as behavioural indicators of declining oxygen saturation during feeding.

\section{Design}

This study was an explorative, secondary analysis of videotapes of preterm infant bottle feeding, which included concurrent oxygen saturation data, in order to learn more about how these infants respond behaviourally to declining oxygen. The original study examined the process of mother-infant co-regulation of bottle feeding prior to discharge from hospital (Thoyre 1997).

\section{Sample}

A convenience sample of 22 very low birthweight infants were observed during a bottle feeding at two Midwestern Neonatal Hospital Units in 1995-1996. All infants had at least 6 days' experience bottle feeding $(15 \cdot 4 \pm 6 \cdot 1,6-24$ days), were capable of oral feeding for at least half of their required feeds over a 24-hour period, and were on average within 1.8 days of full oral feeding ( $\mathrm{sD}=3 \cdot 8$ ). The infants had a range of health prior to the study, with six infants still requiring supplemental oxygen at this time. Excluded from the data collection were infants with anatomical conditions that interfered with feeding, known substance exposure, or significant neurological insults. As behaviour during and prior to oxygen decline was the focus of the analysis, data from two infants were excluded because they had $\mathrm{SpO}_{2}$ levels greater than $90 \%$ throughout their feeding. One infant did not have a single desaturation event during the feeding and the second had desaturation events only during non-feeding periods. Therefore, a total of 20 infant feedings were analysed (see Table 1 for sample characteristics). 


\section{Data collection}

Infants were bottle fed by their mothers in a private parent visiting room according to the nursery's routine. Mothers had bottle fed their infants an average of 14 times prior to the study $(\mathrm{sD}=11.08)$ and anticipated feeding their infants for $80 \%$ of feeds during the month postdischarge. They were asked to feed their infants as they normally would and to indicate when the feed was complete. No contact was made with the mother during the feeding, which was videotaped with the camera angled to capture a close-up of the infant's upper body and head. A small microphone was attached to the rim of the bottle nipple to detect airflow, and the airflow audio signal was transmitted to the videocamera. Infants were monitored with pulse oximetry (Oxicap 3700, Ohmeda, Boulder, CO, USA) set at the shortest averaging time. Mothers had visual access to the infant's $\mathrm{SpO}_{2}$ via the pulse oximeter monitor. The $\mathrm{SpO}_{2}$ signal was transmitted through an analogue-to-digital converter and recorded once per second on a PC computer. Movement artefact was later removed from the $\mathrm{SpO}_{2}$ data by comparing the oximeter pulse rate with the heart rate from a separate cardiorespiratory monitor. An audio tone placed on the videotape and simultaneously marked on the physiological file permitted subsequent alignment of the behavioural and physiological data.

In each infant file a feeding segment of bottle-in-mouth to bottle-removal was selected that was at least 4 minutes in length and included at least one desaturation event. These selected feeding segments were then coded in the laboratory using the OBSERVER behavioural coding program (Version 3.0, Noldus Information Technology Inc., Asheville, NC, USA). A total of 25 behaviours were coded for every second within seven mutually exclusive behavioural classes or as one of three single events. Coded behaviours reflected the quality of movement and muscle tone of the infant's arms, head, eyes and eyebrows; the presence/absence of hiccups, cough, or startle; the presence/absence of milk drooling from mouth; sucking vs. sucking pause; and breath quality (see Table 2 for code definitions). Coding was begun once inter-coder reliability greater than $80 \%$ was achieved for all coded behaviours. Three passes of the data were made during coding: one to code infant arm, head and eye condition, one to code single events, milk drool and sucking, and one to code breath quality. To facilitate coding the quality of each audible breath, the videotape speed was slowed during this pass. Events occurring around periods of noisy breathing and breathing pauses were noted in each file at the time of their occurrence to record the context of breathing difficulties and to generate hypotheses for future study. Following coding of the videotapes, behavioural data and $\mathrm{SpO}_{2}$ data were aligned at one sample per second for analysis.

The occurrences of each behaviour within three oxygen periods (desaturation, immediately preceding desaturations, and high $\mathrm{SpO}_{2}$ ) were compared to identify potential behavioural indicators of oxygen desaturation during feeding. Periods of desaturation were defined as $\mathrm{SpO}_{2}$ levels below $90 \%$ for a minimum of five consecutive seconds, with at least 10 seconds between periods. Predesaturation periods were defined as the 10 -seconds before each desaturation period. High $\mathrm{SpO}_{2}$ periods were defined by $\mathrm{SpO}_{2}$ levels of $95 \%$ or greater for a minimum of five consecutive seconds. High $\mathrm{SpO}_{2}$ periods were identified after the desaturation and predesaturation periods were removed; thus, there was no overlap in time between any events.

\section{Ethical considerations}

The Institutional Review Board of the University of North Carolina approved the study protocol for this secondary analysis. For the original study, the Institutional Review Board of the University of Wisconsin approved the protocol and written informed parental consent was obtained before each study feeding, permitting the data, including the videotapes, to be archived for future research in the area of preterm infant feeding. 


\section{Data analysis}

To indicate the degree to which a behaviour was observed during an oxygen period, the proportion of each oxygen period during which behaviours were observed was calculated. Mean levels of each behaviour for all three oxygen periods were compared using one-way analysis of variance and Tukey's Studentized Range test. This was an exploratory study; therefore an alpha level of 0.10 was used for possible effects, and equal attention was paid to effect sizes.

The concepts of specificity and sensitivity are key to identifying reliable behavioural indicators of oxygen desaturation. A highly sensitive measure of hypoxemia would be an indicator that is likely to occur whenever there is a decline in $\mathrm{SpO}_{2}$. A highly specific measure of hypoxemia would be an indicator that only occurs during periods of $\mathrm{SpO}_{2}$ decline. An ideal indicator of hypoxemia would be both sensitive and specific. For example, an infant would be likely to have a decline in $\mathrm{SpO}_{2}$ during a cyanotic period (sensitive) and would be likely not to have cyanosis during periods of optimal oxygen (specific). However, even cyanosis has been found to be an unreliable measure of hypoxaemia (Comroe \& Botelho 1947, Garg et al. 1988).

Variation in room lighting, skin pigment and haemoglobin level of the infant and inter-observer variation all contribute to assessment error in using cyanosis as a measure of hypoxaemia. In addition, cyanosis is considered a late sign. Under conditions of normal levels of haemoglobin, cyanosis is not detectable until $\mathrm{SpO}_{2}$ declines below 85\% (Moyle 1998). Therefore, cyanosis is not sensitive to declining $\mathrm{SpO}_{2}$ (prehypoxaemia) and is a poor marker of mild hypoxaemia.

For this analysis, all coded behaviours were recoded as being present or absent during desaturation and non-desaturation periods and then tested for specificity and sensitivity in detecting desaturation events using chi-square analysis. A behaviour was considered sensitive if it marked more than $80 \%$ of the desaturation periods and specific if its absence marked more than $80 \%$ of the non-desaturation periods.

\section{Findings}

\section{Feeding segment characteristics}

Coded feeding segments averaged 394 seconds in length ( $S D=85 \cdot 3$, range $=260-575$ seconds). Mean oxygen of coded feeding segments varied by infant (mean $=94 \%, \mathrm{sD}=2 \cdot 1)$. There were brief periods of missing behavioural data because of positioning of the infant or of the blanket or protective cloth, mother's moving in front of the camera or, for the variable of breathing, because of increased room noise. In particular, arms and/or hands out of sight were problematic when mothers placed the protective cloth over the infants' arms. The feeding was not interrupted until the mother removed the nipple; therefore, these periods were at times lengthy. The percentage of missing data for each of the classes of behaviour were: arms/hands, 32.3\%; eyes $1.1 \%$, breathing $7.7 \%$. The events of cough and startle occurred eight and four times, respectively, and hiccups did not occur. These three behaviours were therefore omitted from further analysis.

Table 3 summarizes the percent of time each behaviour, within its class of behaviour, occurred. Each behaviour had a wide range of $\mathrm{SpO}_{2}$ during its occurrence; however, mean $\mathrm{SpO}_{2}$ was similar for all behaviours (mean $=94 \%$, sD 0.53 ).

\section{Characteristics of $\mathrm{SpO}_{2}$ periods}

Descriptions of the $\mathrm{SpO}_{2}$ periods are presented in Table 4. Forty-one desaturation events were identified, ranging from 5 to 101 seconds in length, with $\mathrm{SpO}_{2}$ levels (averaged within each event) ranging from 79.0 to $89.0 \%$. The 10 -second periods prior to these desaturation events denoted the predesaturation events, with averaged $\mathrm{SpO}_{2}$ ranging between $90 \cdot 1$ and $97.0 \%$. 
There were 149 periods of high $\mathrm{SpO}_{2}$, with durations between 5 and 166 seconds and average level of $\mathrm{SpO}_{2}$ ranged from $96 \cdot 0$ to $99 \cdot 3 \%$.

\section{Feeding behaviours}

Within several classes of behaviours, one behaviour dominated across all oxygenation periods. These common feeding behaviours were: arms relaxed, eyes closed soft, eyebrows relaxed, head still, and nipple-in-mouth with no milk drooling out (see Figure 1). The only significant difference among the paired comparisons of the common feeding behaviours between the three oxygenation periods was for arms/hands relaxed, which was 17\% higher during desaturation than during high $\mathrm{SpO}_{2}$.

Of the remaining, less common, feeding behaviours, five significant paired comparisons were found, and are displayed in Figure 2. Apnoea was 16\% higher during predesaturation than during high $\mathrm{SpO}_{2}$ periods and $15 \%$ higher during predesaturation than during desaturation. During predesaturation periods, sucking occurred $18 \%$ more than during desaturation periods, and consequently sucking pauses occurred $17 \%$ more during periods of desaturation than during the predesaturation periods. Several behaviours occurred rarely, that is in less than $15 \%$ of any of the oxygenation periods. These behaviours were: arms flaccid, arms extended, arms move, eye flutter, eyes squeezed, eyebrow raise, and head movement. In these behaviours, only one significant paired comparison was found. Eye flutter occurred $4 \%$ more during predesaturation periods than during high $\mathrm{SpO}_{2}$ periods. All other rarely occurring behaviours occurred with similar proportion during each oxygenation period.

\section{Sensitivity and specificity of behaviours}

None of the behaviours examined was found to be both sensitive and specific for oxygen desaturation. Breathing noisy was a sensitive marker of desaturation, but was not specific. Therefore, when an oxygen desaturation occurred infants were likely to have noisy breathing during that period; however, noisy breathing also occurred during high $\mathrm{SpO}_{2}$ and predesaturation periods during a similar proportion of time. Seven behaviours, all rarely occurring, were specific but not sensitive to desaturation: arms flaccid, arms extending, arms moving, eyes flutter, eyes squeezed, eyebrows raised, and head moving. During nondesaturation periods, it was unlikely for infants to exhibit these behaviours. However, desaturations also occurred in the absence of these behaviours.

\section{Breathing quality}

The breathing quality of quiet, clear airflow was coded during a minority of all three oxygen periods: $21.9 \%$ of high $\mathrm{SpO}_{2}$ periods, $18.8 \%$ of predesaturation, and $26.7 \%$ of desaturation periods. Breathing noisily (airflow either through a partially closed airway or through fluid in the airway) was coded during $44.2 \%$ of high $\mathrm{SpO}_{2}$ periods, $40.5 \%$ of predesaturation periods, and $45.4 \%$ of desaturation periods.

Notes generated during the coding of breath quality identified several recurrent patterns of breathing difficulty. Poor management of fluid and uncoordinated swallowing were identified in 17 of the 20 feedings. All 17 feedings had occurrences of multiple swallowing and breathing changes related to swallowing - multiple swallows prolonged an apnoea episode $(n=15)$, swallowing interrupted breathing bouts leading to an apnoea episode $(n=9)$, or multiple swallows were interspersed with brief, shallow breaths $(n=7)$.

Low energy, as indicated by the infant passively receiving the nipple, not actively engaging in feeding or not actively participating, was common. In several cases, the notation of low energy preceded sonorous breath sounds or an increase in the report of swallowing difficulties. Five 
infants had periods of drowsiness, 12 had periods of sleep, and three were noted to appear exhausted.

\section{Discussion}

The purpose of this exploratory study was to examine whether specific observable behaviours or audible qualities of breathing may show potential as indicators of declining oxygenation during oral feeding. Behaviours were examined during periods of high $\mathrm{SpO}_{2}$, during the 10 seconds immediately prior to oxygen desaturation events, and during desaturation events. The most common overall feeding behaviours were eyes closed softly, eyebrows and head still, arms/hands relaxed, and lips on nipple with no milk drooling.

Arms and/or hands in a relaxed posture occurred significantly more often during desaturation periods than during high $\mathrm{SpO}_{2}$. During high $\mathrm{SpO}_{2}$ periods, infants had more arm/hand movement and arm/hand extension, perhaps reflecting more energy available for movement.

With the exception of eye flutter, less frequent feeding behaviours were not significantly related to declining oxygenation or to a desaturation period. While eye flutter rarely occurred during feeding, when it did occur, this was significantly more often immediately prior to a desaturation period than during a period of high oxygen. However, eye flutter did not consistently occur during all predesaturation periods, and was found to occur on occasion during high oxygen periods. Therefore, while eye flutter may alert the feeder to the infant starting to decline in oxygenation, it is not a sensitive marker of oxygen status.

The proportion of all other behaviours of the eyes, eyebrows, arms and/or hands, and head were not significantly related to oxygen status. In particular, all of the feeding behavioural stress cues described in the literature (eyebrow raise, eyes closed tight, finger splay, arm extension, arm flaccidity, and head movement) (Vandenberg 1990, Als 1998, Shaker 1999) were found to occur during feeding; however, none were temporally related to declining levels of $\mathrm{SpO}_{2}$. While these behaviours tended to not occur during non-desaturation periods, they occurred rarely overall, and did not occur consistently during desaturation periods. Therefore, monitoring for changes in behaviour, particularly these common signs of stress in preterm infants, will not sufficiently detect diminishing $\mathrm{SpO}_{2}$ levels during feeding.

While changes in behaviour may be insufficient to detect $\mathrm{SpO}_{2}$ decline, commonly cited behavioural stress cues may still be indicative of feeding distress. Additional behavioural research is needed to explore the relationship of commonly cited stress cues during feeding with feeding problems, such as swallowing dysfunction. For example, when milk flow exceeds the infant's capacity for swallowing efficiently, the infant may indicate distress by elevating the eyebrows, wrinkling the forehead, or pulling the head away from the nipple. Oxygenation may remain stable, but the infant may still be experiencing a threat to the airway and thus may need an intervention.

The most significant markers of oxygen decline during feeding were the presence or absence of sucking and incidence of breathing pauses greater than 4 seconds (defined as apnoea). Consistent with Shiao et al. (1996), immediately prior to desaturation events infants were more often found to be apnoeic and/or sucking. During desaturation events they stopped sucking and breathing normalized. As infants tend to stop sucking when their oxygenation declines, sucking pauses may represent an attempt to self-regulate oxygenation. Caregivers can support infants in re-regulating their breathing pattern during sucking pauses by encouraging a period of breathing and avoiding movement of the bottle nipple to stimulate sucking.

The breathing pauses examined were brief; yet even brief apnoea was an important indicator of desaturation during feeding. Repeated, brief apnoea during feeding is likely to increase the 
risk of hypoxaemia. During non-feeding periods, Poets et al. (1997b) found that apnoeic pauses resulted in a significant reduction in the amount of air remaining in the lungs after expiration, which increased the preterm's vulnerability to hypoxaemia even with relatively short breathing pauses. Individual infants are likely to tolerate different lengths of breathing pauses during feeding, based on their baseline $\mathrm{SpO}_{2}$, postconceptional age, history of respiratory health, and prefeeding respiratory rate (Shiao et al. 1996). Therefore, during feeding a subgroup of preterm infants will be sensitive to even minor changes in their pattern of breathing. This vulnerability may contribute to the prolonged transition period to full oral feeding found in preterm infants who continue to have apnoea between feeds (Mandich et al. 1996).

\section{What is already known about this topic}

- Episodes of oxygen decline are a significant complication of bottle feeding for preterm infants.

- Nurses and families are advised to monitor preterm infants for signs of stress during oral feeding.

\section{What this paper adds}

- Breathing and sucking patterns will need to be monitored during bottle feeding to sensitively feed the preterm.

- During bottle feeding some infants will be sensitive to even minor changes in their breathing pattern leading to oxygen decline.

- Movement of the bottle nipple during preterm infant sucking pauses may contribute to oxygen dysregulation.

- Monitoring for common signs of stress in the preterm will not sufficiently detect diminishing oxygen saturation during bottle feeding.

Further study is needed to predict which infants are more vulnerable to changes in breathing pattern and what types of support are required to assist in maintaining physiological stability during feeding. Caregivers will need to determine, with the aid of pulse oximetry, what length of breathing pause an individual infant can tolerate and at what point the infant begins to decline in $\mathrm{SpO}_{2}$. The infant can then be cued to stop sucking and re-commence breathing, termed pacing, by moving the nipple to the roof of the mouth or by full removal of the nipple. Some infants will continue to suck if the nipple remains in their mouth, responding to its stimulation; therefore, the caregiver will need to determine the infant's sensitivity to various pacing techniques.

Not only is the presence or absence of breathing significant, but the quality of the breathing is important. Brief, shallow breathing has been found to lead to persistent reduction in functional residual capacity (Poets et al. 1997b) and loss of oxygen reserves (Henderson-Smart 1980,

Henderson-Smart et al. 1986). Therefore, in addition to breathing pauses, inadequate depth of breath during sucking and swallowing phases of feeding may lower minute ventilation, leading to less tolerance for even brief breathing pauses. In addition, infants who become fatigued or fall asleep during feeding may experience loss of muscle activity in the intercostals and upper airway-maintaining musculature similar to that which occurs during active sleep (Brouillette \& Côté 1996). Noisy breathing may be an indicator of decreased pharyngeal, airwaymaintaining muscular activity. This potential for decreased muscle tone in the upper airway during feeding warrants further study.

Notes made during the breath coding confirm the need to explore the coordination and efficiency of swallowing, context and impact of laboured breathing, and impact of fatigue on 
preterm infant feeding skills. As these infants inefficiently integrate swallowing and breathing (Wilson et al. 1981, Hanlon et al. 1997), the relationship of swallowing apnea to hypoxaemia during feeding needs further study.

The impact of fatigue during feeding on the capacity to respond behaviourally to declining oxygen levels, to swallow efficiently, and to maintain smooth regulation of respirations has not been adequately explored and needs further research. Infants are reliant on caregivers to interpret accurately their physiological state and to co-regulate their physiological stability.

However, preterm infants, who are prone to fatigue during feeding, may not reliably indicate distress during feeding when their energy levels decline. Once an infant's capacity to respond diminishes, they become increasingly vulnerable to undetected desaturation events. In our sample, those identified as sleepy or fatigued typically gave few behavioural cues of significantly diminished oxygenation other than a change in the quality of breathing or the absence of sucking. A larger sample size is required to test this hypothesis formally.

\section{Study limitations}

Examining single behavioural clinical indicators of desaturation may provide an incomplete picture of the patterns of behaviours that may occur during oxygen desaturation. The method used in this study would be enhanced by examining the occurrence of multiple behavioural predictors that may identify transitions to desaturation events using the entire feeding as the unit of analysis with a larger sample size.

\section{Conclusion}

The most compelling cue to impending decline in $\mathrm{SpO}_{2}$ during oral feeding was the occurrence of a breathing pause and a change in sucking. Behaviours that are considered to be preterm infant stress cues occurred rarely during feeding and, compared with periods of high $\mathrm{SpO}_{2}$, were not more likely to occur as oxygen was declining or during oxygen desaturation. Therefore, behaviour may be an unreliable means of detecting oxygen desaturation during feeding. As feeding requires effort and energy expenditure, the infant's capacity for a behavioural response to declining oxygenation may diminish as they become fatigued. In addition, ability to tolerate even brief apnoea and the capacity to manage the milk flow from the bottle may change through the course of a feed as the infant's state, oxygen reserves, and energy level are affected by the activity of feeding. Interventions that focus on minimization of apnoea, support of adequate depth of breathing, prevention of fatigue, and protection of selfregulatory mechanisms may influence the number and severity of desaturation events experienced by preterm infants.

\section{Acknowledgments}

This study was supported by the University of North Carolina at Chapel Hill School of Nursing and the National Institute of Nursing Research, Fellowship NR07052-02. Special thanks are extended to the mothers and infants who participated in the study, to John L. Doyne Hospital of Milwaukee WI and Meriter Hospital of Madison WI, to Autumn Thoyre, Laura Maddox, and Chris Caleffie for coding the videotapes of the infant feedings, and to Diane HolditchDavis for review of the manuscript.

\section{References}

Als H. Developmental care in the newborn intensive care unit. Current Opinion in Pediatrics 1998;10:138-142. [PubMed: 9608890]

Brouillette, RT.; Côé, A. Physiologic control of respiration. In: Gluckman, PD.; Heymann, MA., editors. Pediatrics and Perinatology: The Scientific Basis. 2nd edn. Arnold; New York: 1996. p. 832-836. 
Bu'Lock F, Woolridge MW, Baum JD. Development of co-ordination of sucking, swallowing and breathing: ultrasound study of term and preterm infants. Developmental Medicine and Child Neurology 1990;32:669-678. [PubMed: 2210082]

Case-Smith J, Cooper P, Scala V. Feeding efficiency of premature neonates. The American Journal of Occupational Therapy 1989;43:245-250. [PubMed: 2750853]

Chen C, Wang T, Chang H, Chi C. The effect of breast- and bottle-feeding on oxygen saturation and body temperature in preterm infants. Journal of Human Lactation 2000;16:21-27. [PubMed: 11138220]

Cheung PY, Barrington KJ, Finer NN, Robertson CM. Early childhood neurodevelopment in very low birth weight infants with predischarge apnea. Pediatric Pulmonology 1999;27:14-20. [PubMed: 10023786]

Comroe JH, Botelho S. The unreliability of cyanosis in the recognition of arterial anoxemia. American Journal of Medicine and Science 1947;214:1-6.

Craig KD, Whitfield MF, Grunau RVE, Linton J, Hadjistavropoulos HD. Pain in the preterm neonate: behavioural and physiological indices. Pain 1993;52:287-299. [PubMed: 8460047]

Craig CM, Lee DN, Freer YN, Laing IA. Modulations in breathing patterns during intermittent feeding in term infants with bronchopulmonary dysplasia. Developmental Medicine and Child Neurology 1999;41:616-624. [PubMed: 10503920]

Garg M, Kurzner SI, Bautista DB, Keens TG. Clinically unsuspected hypoxia during sleep and feeding in infants with bronchopulmonary dysplasia. Pediatrics 1988;81:635-642. [PubMed: 3357725]

Grunau RE, Craig KD. Pain expression in neonates: facial action and cry. Pain 1987;28:395-410. [PubMed: 3574966]

Grunau RE, Holsti L, Whitfield MF, Ling E. Are twitches, startles, and body movements pain indicators in extremely low birth weight infants? Clinical Journal of Pain 2000;16:37-45. [PubMed: 10741817]

Hanlon MB, Tripp JH, Ellis RE, Flack FC, Selley WG, Shoesmith HJ. Deglutition of apnoea as indicator of maturation of suckle feeding in bottle-fed preterm infants. Developmental Medicine and Child Neurology 1997;39:534-542. [PubMed: 9295849]

Henderson-Smart DJ. Vulnerability to hypoxemia in the newborn. Sleep 1980;3:331-342. [PubMed: 7221342]

Henderson-Smart DJ, Butcher-Puech MC, Edwards DA. Incidence and mechanism of bradycardia during apnoea in preterm infants. Archives of Disease in Childhood 1986;61:227-232. [PubMed: 3963865]

Hill A. Preliminary findings: a maximum oral feeding time for premature infants, the relationship to physiological indicators. Maternal-Child Nursing Journal 1992;20:81-92. [PubMed: 1287371]

Hill A, Rath L. The relationship between drooling, age, sucking pattern characteristics and physiological parameters of preterm infants during bottle feeding. Research for Nursing Practice 1999;1 retrieved 30 December 2002, from http://www.graduateresearch.com/hill.htm.

Hill AS, Kurkowski TB, Garcia J. Oral support measures used in feeding the preterm infant. Nursing Research 2000;49:2-10. [PubMed: 10667623]

Holditch-Davis D. Behaviors of pretermn infants with and without chronic lung disease when alone and when with nurses. Neonatal Network 1995;14:51-57. [PubMed: 7565527]

Holditch-Davis, D.; Lee, DA. The behaviors and nursing care of preterm infants with chronic lung disease. In: Funk, SG.; Tornquist, EM.; Champayne, MT.; Wiese, RA., editors. Key Aspects of Caring for the Chronically Ill: Hospital and Home. Springer; New York: 1993. p. 250-270.

Johnston CC, Stevens B, Yang F, Horton L. Developmental changes in response to heelstick in preterm infants: a prospective cohort study. Developmental Medicine and Child Neurology 1996;38:438445. [PubMed: 8698151]

Kaye K, Wells AJ. Mother's jiggling and the burst-pause pattern of neonatal feeding. Infant Behavior and Development 1980;3:29-46.

McGrath JM, Medoff-Cooper B. Alertness and feeding competence in extremely early born preterm infants. Newborn and Infant Nursing Reviews 2002;2:174-186.

Mandich MB, Ritchie SK, Mullett M. Transition times to oral feeding in premature infants with and without apnea. Journal of Obstetric, Gynecologic, and Neonatal Nursing 1996;25:771-776.

Mathew OP. Science of bottle feeding. Journal of Pediatrics 1991;119:511-519. [PubMed: 1919879] 
Mathew OP, Clark ML, Pronske ML, Luna-Solarzano HG, Peterson MD. Breathing pattern and ventilation during oral feeding in term newborn infants. Journal of Pediatrics 1985;106:810-813. [PubMed: 3998923]

Moyer-Mileur LJ, Nielson DW, Pfeffer KD, Witte MK, Chapman DL. Eliminating sleep-associated hypoxemia improves growth in infants with bronchopulmonary dysplasia. Pediatrics 1996;98:779783. [PubMed: 8885961]

Moyle, JTB. Pulse oximetry. Rev. edn.. BMJ Publishing Group; London: 1998.

Perlman JM. Neurobehavioral deficits in premature graduates of intensive care - potential medical and neonatal environmental risk factors. Pediatrics 2001;108:1339-1348. [PubMed: 11731657]

Peters K. Association between autonomic and motoric systems in the preterm infant. Clinical Nursing Research 2001;10:82-91. [PubMed: 11881754]

Poets CF, Langner MU, Bohnhorst B. Effects of bottle feeding and two different methods of gavage feeding on oxygenation and breathing patterns in preterm infants. Acta Paediatrica 1997a;86:419423. [PubMed: 9174231]

Poets CF, Rau GA, Neuber K, Gappa M, Seidenberg J. Determinants of lung volume in spontaneously breathing preterm infants. American Journal of Respiratory and Critical Care Medicine 1997b; 155:649-653. [PubMed: 9032208]

Porges $\mathrm{S}$. The infant's sixth sense: awareness and regulation of bodily processes. Zero to Three 1994;14:12-16.

Rosen CL, Glaze DG, Frost JD. Hypoxemia associated with feeding in the preterm infant and full-term neonate. American Journal of Disease in Childhood 1984;138:623-628.

Shaker CS. Nipple feeding preterm infants: an individualized, developmentally supportive approach. Neonatal Network 1999;18:15-22. [PubMed: 10418433]

Shiao SY. Comparison of continuous versus intermittent sucking in very-low-birth-weight infants. Journal of Obstetric, gynecological, and Neonatal Nursing 1997;26:313-319.

Shiao SY, Brooker J, DiFiore T. Desaturation events during oral feedings with and without a nasogastric tube in very low birth weight infants. Heart and Lung 1996;25:236-245. [PubMed: 8635924]

Shivpuri CR, Martin RJ, Carlo WA, Fanaroff AA. Decreased ventilation in preterm infants during oral feeding. Journal of Pediatrics 1983;103:285-289. [PubMed: 6875726]

Singer L, Martin RJ, Hawkins SW, Benson-Szekely LJ, Yamashita TS, Carlo WA. Oxygen desaturation complicates feeding in infants with bronchopulmonary dysplasia after discharge. Pediatrics 1992;90:380-384. [PubMed: 1518692]

Thoyre, S. Co-regulation in preterm infant feeding. University of Wisconsin; Madison, WI: 1997. Unpublished Doctoral Dissertation

Vandenberg KA. Nippling management of the sick neonate in the NICU: the disorganized feeder. Neonatal Network 1990;9:9-16. [PubMed: 2381414]

Wilson SL, Thach BT, Brouillette RT, Abu-Osba YK. Coordination of breathing and swallowing in human infants. Journal of Applied Physiology 1981;50:851-858. [PubMed: 7263368] 


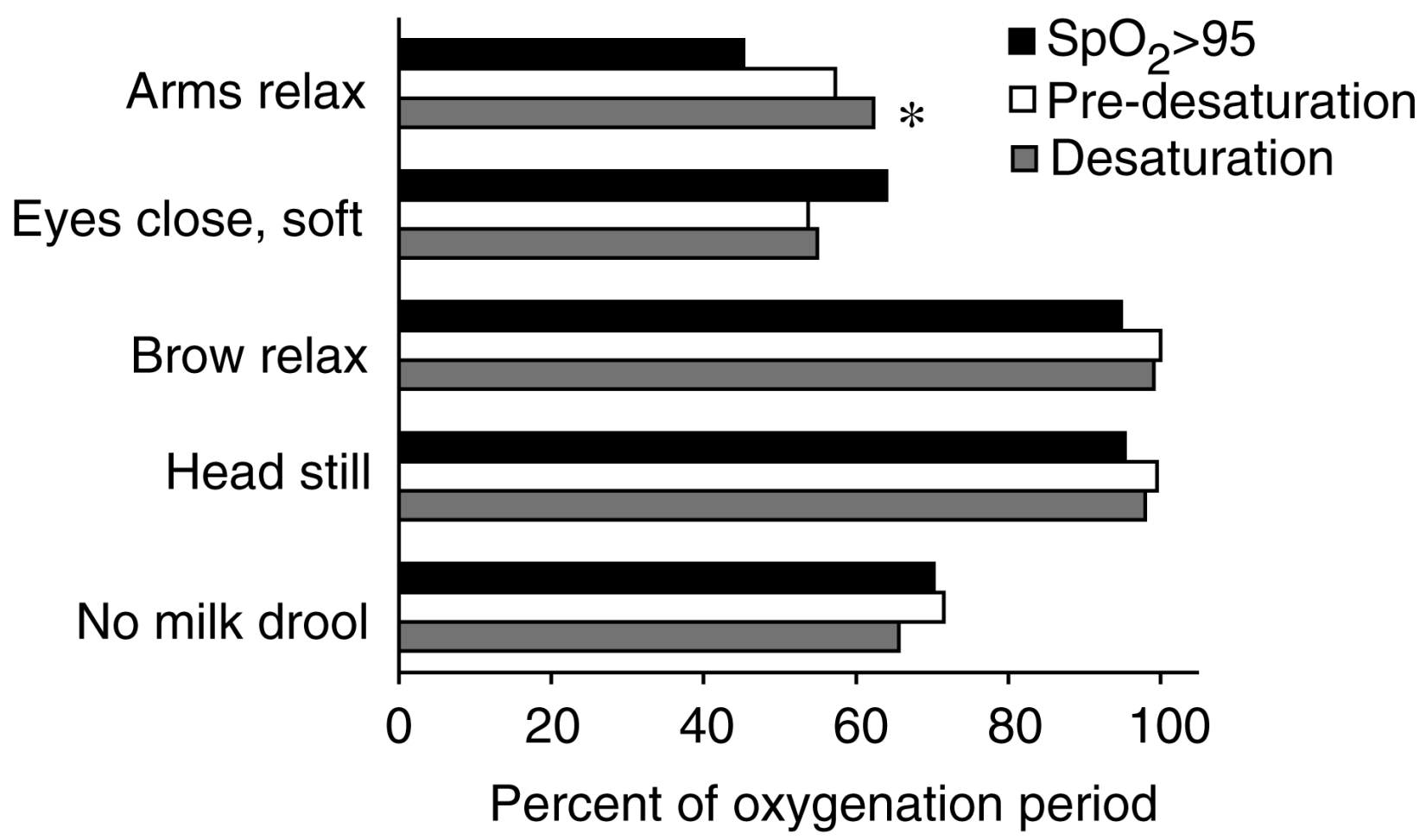

Figure 1.

Common feeding behaviours during three oxygenation periods. *Significant differences (Tukeys Standardized Range Test, $P<0 \cdot 10$ ) in proportion of behaviours. 


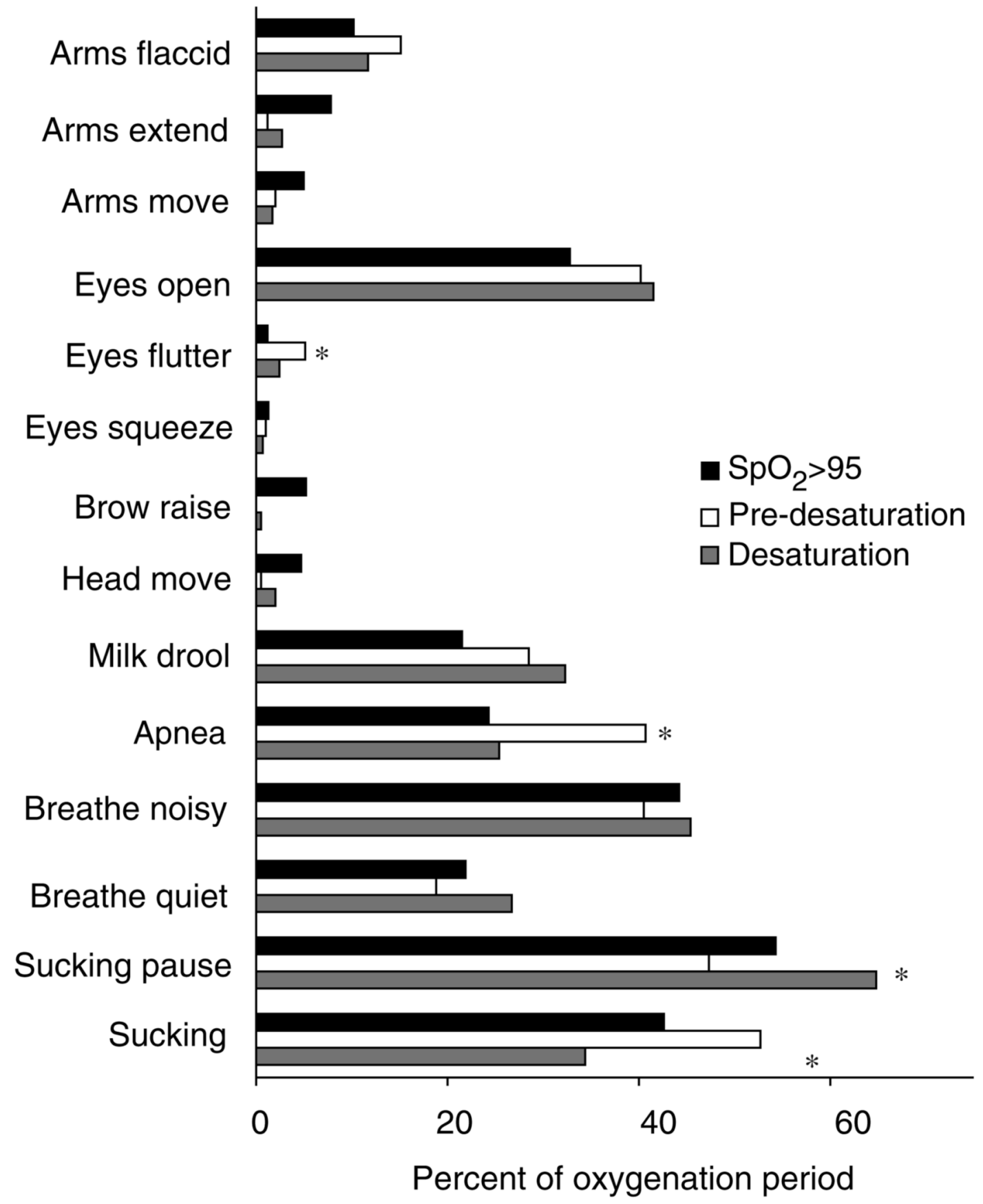

Figure 2.

Potential clinical indicators of feeding desaturation. *Significant differences (Tukeys Standardized Range Test, $P<0 \cdot 10$ ) in proportion of behaviours between three oxygenation periods: high $\mathrm{SpO}_{2}$, predesaturation, and desaturation. 


\section{Table 1}

Description of study participants $(n=20)$

\begin{tabular}{lcc}
\hline Variable & Mean \pm SD & Range \\
\hline Gestational age at birth & $28 \cdot 2 \pm 2 \cdot 16$ & $25-32$ weeks \\
Postconceptional age at study & $36 \cdot 5 \pm 1 \cdot 68$ & $33 \cdot 5-40$ weeks \\
Weight, grams at birth & $1172 \pm 301 \cdot 1$ & $620-1500 \mathrm{~g}$ \\
Weight, grams at study & $2184 \pm 315$ & $1692-2720 \mathrm{~g}$ \\
Days ventilated & $6 \cdot 3 \pm 12 \cdot 8$ days \\
Days CPAP & $12 \cdot 4 \pm 16 \cdot 4$ & $0-54$ days \\
Duration of supplemental $\mathrm{O}_{2}$, days & $38 \pm 32 \cdot 7$ & $1-97$ days \\
Per cent on supplemental $\mathrm{O}_{2}$ at study & $30 \%$ & $90-98 \%$ \\
Baseline $\mathrm{SpO}_{2}$ prior to study & $93 \pm 2 \cdot 6$ & \\
Gender & $40 \%$ Male & \\
& $60 \%$ Female & \\
Race & $70 \%$ Caucasian & \\
\hline
\end{tabular}


Table 2

\title{
Behavioural code definitions
}

\author{
Mutually exclusive behavioural classes \\ Arms and/or hands
}

Relaxed: Arms and/or hands relaxed, normal muscle tone, not moving, flexing, or extending; there is visible muscle tone in the arm/hand as evidenced by arm/hand not lying flaccidly.

Extending: Arms and/or hands extended, fingers may be splayed outward or arm may be stretched outward or pushing outward.

Moving: Arms and/or hands moving.

Flaccid: Arms and/or hands flaccid, hanging loosely, droopy.

Eyes

Open: Eyes open.

Closed, soft: Eyes closed softly as if asleep.

Closed, squeezed: Eyes closed tightly, scrunched, squeezing eyes shut.

Flutter: Less than one second between blinks (rapid back and forth eye closing and re-opening), or repeated eye opening and closing.

Eyebrows

Relaxed: Eyebrows are relaxed, not raised.

Raised: Eyebrows are raised.

Head

Still: Head is still, not moving.

Moving: Head is moving - helping to search for nipple, moving toward nipple in acceptance or head is avoiding the nipple; may pull away, move side to side, or push forward.

Bottle-in-mouth

No milk drooling out while nipple is completely seated in the mouth.

Milk drooling out while nipple is completely seated in the mouth.

Breath quality

Quiet: Breath sounds are quiet, normal, easy, non-laboured; may be shallow or deep, fast or slow; there is a clear sound to the passage of air (no obstruction with fluid or constriction).

Noisy: Breath sounds are noisy. There is obstruction to the free flow of air. They may be grunty sounding; a dry, coarse sound; no fluid in airway; child may be pushing air outward noisily; may be breathing rapidly or slowly as in a snore; a sound of constriction at the back of the throat. A non-laboured whistle alone would not be noisy breathing. Whistle plus some indication of laboured breathing is noisy breathing (a fluid 'catch' sound, a clear obstruction of airflow, or gurgly breath). Alternatively, the noisy breathing may be a wet, gurgly sound; fluid in the child's airway (nasal or throat) that air is audible passing over or through. May be a sound of a fluid 'catch' at the end of inspiration or expiration.

Absent for at least 4 seconds (apnea). During a time when breath sounds are audible you detect no breathing for $\geq 4$ seconds.

Sucking.

Pausing: Period of no sucking.

Single behavioural events

Hiccups.

Cough.

Startle. 
Table 3

Frequency of feeding behaviour occurrence and oxygen saturation for each behaviour $\left(\% \mathrm{SpO}_{2}\right)$

\begin{tabular}{|c|c|c|c|}
\hline \multirow[t]{2}{*}{ Behavioural codes } & \multirow{2}{*}{$\begin{array}{l}\text { Frequency } \\
\text { (percentage } \\
\text { of segment) }\end{array}$} & \multicolumn{2}{|l|}{$\mathrm{SpO}_{2}$} \\
\hline & & Mean \pm SD & Range \\
\hline \multicolumn{4}{|l|}{ Arms and/or hands $*$} \\
\hline Relaxed & 43.6 & $93 \cdot 6 \pm 4 \cdot 3$ & $65-100$ \\
\hline Extending & $6 \cdot 1$ & $95 \cdot 0 \pm 2 \cdot 86$ & $86-100$ \\
\hline Moving & $5 \cdot 4$ & $93 \cdot 4 \pm 3.41$ & $63-99$ \\
\hline Flaccid & $12 \cdot 6$ & $93 \cdot 1 \pm 2 \cdot 96$ & $78-99$ \\
\hline \multicolumn{4}{|l|}{ Eyes $^{\dagger}$} \\
\hline Open & $33 \cdot 1$ & $94 \cdot 2 \pm 3 \cdot 38$ & $78-100$ \\
\hline Closed, soft & $63 \cdot 2$ & $93 \cdot 9 \pm 4 \cdot 16$ & $71-100$ \\
\hline Closed, squeezed & 0.7 & $93 \cdot 7 \pm 4 \cdot 12$ & $78-97$ \\
\hline Flutter & 1.9 & $94 \cdot 3 \pm 2 \cdot 97$ & $86-99$ \\
\hline \multicolumn{4}{|l|}{ Eyebrows } \\
\hline Relaxed & 96 & $94.0 \pm 3.91$ & $71-100$ \\
\hline Raised & 4 & $95 \cdot 2 \pm 2 \cdot 63$ & 79-99 \\
\hline \multicolumn{4}{|l|}{ Head } \\
\hline Still & $95 \cdot 3$ & $94.0 \pm 3.91$ & $65-100$ \\
\hline Moving & $4 \cdot 7$ & $93 \cdot 4 \pm 4 \cdot 14$ & $63-100$ \\
\hline \multicolumn{4}{|l|}{ Bottle-in-mouth ${ }^{f}$} \\
\hline No milk drooling & 68 & $94 \cdot 1 \pm 3 \cdot 85$ & $65-100$ \\
\hline Milk drooling out & $25 \cdot 6$ & $94 \cdot 0 \pm 4 \cdot 11$ & $76-100$ \\
\hline \multicolumn{4}{|l|}{ Breath quality $\S$} \\
\hline Quiet & 24 & $94.4 \pm 3.93$ & $71-100$ \\
\hline Noisy & $50 \cdot 3$ & $94 \cdot 0 \pm 3.83$ & $65-100$ \\
\hline Absent for at least 4 seconds (apnea) & 18 & $93 \cdot 8 \pm 4 \cdot 15$ & $71-100$ \\
\hline Sucking $I I$ & $43 \cdot 1$ & $94 \cdot 3 \pm 3 \cdot 53$ & $72-100$ \\
\hline Pausing & 54 & $93 \cdot 8 \pm 4 \cdot 17$ & $65-100$ \\
\hline \multicolumn{4}{|c|}{ * Arms and/or hands were not visible $32.3 \%$ of the coding period. } \\
\hline \multicolumn{4}{|l|}{${ }^{t}$ Eyes out of sight $1.1 \%$ of the coding period. } \\
\hline
\end{tabular}


Table 4

Description of $\mathrm{SpO}_{2}$ during each oxygenation study period

\begin{tabular}{lrr}
\hline Oxygenation period & Mean \pm sD & Range \\
\hline High saturation $(n=149)$ & $96 \cdot 9 \pm 0.76$ & $96-99 \cdot 3 \%$ \\
Predesaturation $(n=41)$ & $93 \cdot 2 \pm 1 \cdot 79$ & $90 \cdot 1-97 \%$ \\
Desaturation $(n=41)$ & $85.99 \pm 2.43$ & $79-89 \%$ \\
\hline
\end{tabular}

